\section{Ochrobactrum anthropi septic arthritis: case report and implications in orthopedic infections}

\author{
Carla C. Saveli, Marilyn Levi, \\ John Koeppe \\ University of Colorado Denver, \\ Department of Medicine, Division of \\ Infectious Diseases, USA
}

\section{Abstract}

Ochrobactrum anthropi is a rare cause of orthopedic infections. We report the second case of Ochrobactrum anthropi septic arthritis in the literature. Our case highlights the ability of Ochrobactrum anthropi to cause septic arthritis and its relevance in the field of orthopedic infections.

\section{Introduction}

Ochrobactrum anthropi (O. anthropi) is a gram negative bacillus widely distributed in the environment and rarely implicated as a human pathogen. ${ }^{1}$ This opportunistic bacterium has been reported in clinical infections in immunocompetent and immunocompromised hosts $^{24}$ but none has addressed its relevance and treatment challenges in bone and joint infections. We report a case of successfully treated 0 . anthropi septic arthritis, and its implication in the orthopedic infections field.

\section{Case Report}

A 53-year old man with history of gout, alcoholism and right knee anterior and posterior cruciate ligament repair presented to a local hospital with complaints of right knee and right wrist pain. Four days prior to presentation he experienced a fall while intoxicated resulting in skin abrasion of his right knee and trauma of both his right knee and wrist. On exam, his right knee had an abrasion just over the tibial tubercle, moderate effusion, increased warmth and erythema. The right wrist evaluation revealed significant erythema and increased warmth. Laboratory findings revealed a white blood cell count of $12,800 / \mathrm{mL}$, elevated C-reactive protein at $33.8 \mathrm{mg} / \mathrm{dL}$ and elevated erythrocyte sedimentation rate at 35 $\mathrm{mm}$. A right knee arthrocentesis performed in the Emergency Department showed a white cell count of 2,250,000/MM with $90 \%$ polymorphonuclear cells and gram positive cocci present on gram stain. He was started empirically on treatment with intravenous vancomycin and levofloxacin. The patient underwent urgent irrigation and debridement of his knee with evidence of frank purulence. An intraoperative right radiocarpal arthrocentesis revealed purulent material with polymorphonuclear white cells and gram positive cocci present on gram stain. An open right radiocarpal irrigation and debridement followed the knee intervention. Synovial fluid culture from his knee grew Group G Beta Hemolytic Streptococci and gram negative rods later identified as 0 . anthropi. Synovial fluid cultures from his wrist yielded Group G Beta Hemolytic Streptococci. Blood cultures obtained 24 hours after the surgical intervention yielded negative results; however, these were obtained after the administration of antibiotics. Input from the Infectious Disease service was sought, who recommended the addition of trimethoprimsulfamethoxazole and ceftriaxone to his antibiotic regimen. The patient underwent two more irrigation and debridements of the right wrist and one more of the right knee. The right knee fluid cultures remained negative after the first intervention. In the light of persistent positive cultures of his radiocarpal joint, his care was transferred to the University of Colorado Hospital. On presentation to our institution his temperature was 39.3. His right knee had a longitudinal incision with sutures, a moderate effusion and no evidence of discharge. His right wrist had a surgical incision with sutures and no evidence of discharge. A systolic heart murmur not mentioned on previous evaluations led to the work-up of endocarditis with negative blood cultures and transthoracic echocardiogram. Two additional right knee arthrocentesis revealed calcium pyrophosphate and monosodium urate crystals but negative gram stain and cultures.

At the referring hospital, the knee synovial fluid culture yielded gram negative, motile, oxidase positive bacillus subsequently identified as 0 . anthropi by inoculation on VITEK ${ }^{\circledR}$ ID-GNB card (bioMérieux Inc., Durham, NC, USA). To exclude the possibility of misidentification of Brucella spp as Ochrobactrum, negative serum Brucella spp antibodies was confirmed in our patient. Minimal Inhibitory Concentration (MIC) testing to the most commonly used drugs reported in the literature was performed by Etest: $2 \mu \mathrm{g} / \mathrm{mL}$ for gentamicin, $1 \mu \mathrm{g} / \mathrm{mL}$ for imipenem, $0.12 \mu \mathrm{g} / \mathrm{mL}$ for levofloxacin and $0.06 \mu \mathrm{g} / \mathrm{mL}$ for trimethoprimsulfamethoxazole. Based on the lowest MIC, oral trimethoprim-sulfamethoxazole $800 \mathrm{mg}$ $160 \mathrm{mg} 2$ tablets every 12 hours was elected for treatment of the 0 . anthropi and intravenous ceftriaxone $2 \mathrm{~g}$ daily for Group G Beta Hemolytic Streptococci. Rheumatology con-
Correspondence: Carla C. Saveli, University of Colorado Denver, Division of Infectious Diseases 12700 E. 19th Avenue, B-168, Aurora, C0 80045,USA. E-mail. carla.saveli@ucdenver.edu

Key words: Ochrobactrum anthropi, septic arthritis, osteomyelitis.

Contributions: CCS, ML and JK have contributed equally to the writing of this manuscript. The final manuscript has been reviewed and approved by all the authors. The manuscript has not been submitted to and is not being considered for publication in another journal.

Conflict of interest: the authors report no conflicts of interest.

Received for publication: 10 September 2009. Revision received: 21 December 2009.

Accepted for publication: 30 December 2009.

This work is licensed under a Creative Commons Attribution 3.0 License (by-nc 3.0).

(C) Copyright C.C. Saveli et al., 2010

Licensee PAGEPress, Italy

Infectious Disease Reports 2010; 2:e2

doi:10.4081/idr.2010.e2

sultants recommended a steroid taper for treatment of acute gout and pseudogout. The patient remained afebrile for 72 hours with significant improvement of his symptoms. He was discharged on a four week course of antibiotic therapy. One year after admission, he is without evidence of recurrent infection.

\section{Discussion}

O. anthropi is a non-fermentative, strictly aerobic, motile, oxidase positive and indole negative, Gram negative bacillus of low virulence that occasionally causes human infection. Formerly known as Center for Disease Control and Prevention (CDC) group Vd, the genus Ochrobactrum and its type species $O$. anthropi were described by Holmes et al. in 1988. 'The Greek word "Ochros" meaning "pale yellow" describes the appearance of its colonies on agar. Ubiquitous in soil and water, $O$. anthropi has also been recovered from contaminated biologic products, ${ }^{2}$ hospital environments, graft tissue, ${ }^{3}$ intravascular catheters, foreign bodies and clinical specimens including: blood, urine, stools, wounds, bile, throat and vagina. ${ }^{4,5}$ Since it is closely related to Brucella spp., 0 . anthropi misidentification can occur with some of the automated systems such as API 20NE. ${ }^{6}$ As a result, confirmation with negative serum Brucella spp. antibodies is recommended in patients with severe disease manifested primarily as 0 . anthropi bacteremia with no obvious 
focus of infection and refractory to standard treatment. The first case of human infection with 0 . anthropi was described in 1980 in a debilitated patient with a pancreatic abscess. ${ }^{7}$ Since then, 0 . anthropi has been infrequently reported as a cause of human infection, mostly associated with nosocomial acquisition, indwelling catheters, retained foreign bodies and hosts with impaired immune function. ${ }^{8}$ Predisposing conditions for infection by this organism include the presence of indwelling medical devices, previous antibiotic therapy, a prior surgical procedure with allografts, traumatic wounds, coinfection with another bacterium, and impaired host immunity. ${ }^{9}$ Published reports suggest that $\boldsymbol{O}$. anthropi is an emerging pathogen in immunocompetent patients and orthopedic infections caused by this organism may be increasing in frequency. To date, one case of septic arthritis and 2 cases of osteomyelitis have been reported in the medical literature (Table 1). A Medline search of the terms Ochrobactrum anthropi, CDC group Vd, arthritis and osteomyelitis from 1950 to the present yielded 4 citations. A review of these 4 cases plus our own indicates that this pathogen is acquired either through direct inoculation from the environment ${ }^{10,11}$ or through hematogenous dissemination. ${ }^{12,13}$

To the best of our knowledge, this is the second reported case of septic arthritis due to $O$. anthropi. In the case presented here, the presumed source of infection is a traumatic wound which served as a port of entry for both $O$. anthropi and Group G Beta Hemolytic Streptococci. The presence of associated bacteremia is unknown since blood cultures were obtained after the administration of antimicrobial therapy. It is interesting that only the knee fluid culture grew both Group G Beta Hemolytic Streptococci and 0 . anthropi, whereas the wrist fluid culture grew only the former organism. We consider posttraumatic right knee septic arthritis as his primary diagnosis presumably associated with Streptococci bacteremia that seeded his right wrist. Since $\boldsymbol{O}$. anthropi has been described as a bacterium with low virulence and low pathogenicity, ${ }^{6}$ it may have caused only localized infection to the right knee joint.

A particular characteristic of this organism is the ability to adhere to foreign bodies. This was first evaluated by Alnor et al. who noted that patients with 0 . anthropi bacteremia did not recover until their catheters had been removed. They found in vitro that the binding ability of $O$. anthropi to silicone tubes was similar to that of Staphylococcus aureus and Staphylococcus epidermidis. ${ }^{5}$ This is relevant for hardware associated orthopedic infections where organisms with the ability to adhere to foreign bodies by the formation of biofilm are much more difficult to cure without radical debridement of the tissue, including hardware removal. Fortunately, the patient in this case had three previous

Table 1. Demographics, treatment and outcomes of reported cases of $O$. anthropi septic arthritis and osteomyelitis.

\begin{tabular}{lcccllll} 
REF* Year & $\begin{array}{c}\text { Age } \\
\text { (yrs) }\end{array}$ & Sex & Diagnosis & $\begin{array}{l}\text { Contributing } \\
\text { condition }\end{array}$ & Treatment & Outcome \\
\hline 11 & 1987 & 14 & M & Osteochondritis & Trauma & CLI/RIF & Cure \\
12 & 1999 & 2 & M & Osteomyelitis & Unknown & CLI/RIF & Cure \\
\hline 13 & 2002 & 62 & F & Osteomyelitis & Bacteremia & CFT/CIP & Cure \\
${ }^{\text {PRR }}$ & 2007 & 53 & M & Septic arthritis & Trauma & COT & Cure \\
\hline 10 & 2008 & 17 & M & Septic arthritis & Trauma & CIP/COT & Cure \\
\hline
\end{tabular}

*REF, reference. †CLI, clindamycin; RIF, rifampin; CFT, ceftriaxone; CIP, ciprofloxacin; COT, trimethoprim-sulfamethoxazole.

interventions in the affected knee joint with no placement of foreign material. Treatment options for 0 . anthropi are limited. This bacterium is inherently resistant to all beta-lactams (penicillins, cephalosporins and aztreonam) except imipenem which is consistent with the expression of an inducible AmpC $\beta$-lactamase. ${ }^{14}$ A study performed to Teyssier et al. performed in vitro antibiotic susceptibility testing of 21 strains of 0 . anthropi. They observed general susceptibility of the strains to gentamicin, tobramycin, rifampin, fluoroquinolones, netilmicin, colistin and trimethprim-sulfamethoxazole. ${ }^{15}$ Nonetheless, clinical failures with imipenem ${ }^{16}$ and ciprofloxacin ${ }^{17}$ therapy despite in vitro susceptibility have been reported. In summary, we have presented a case of $\boldsymbol{O}$. anthropi septic arthritis in an adult. To the best of our knowledge, this is the second such report. Our case highlights the ability of $O$. anthrop $i$ to cause septic arthritis and its relevance in the field of orthopedic infections. Treatment options are limited and selection of the proper agent is critical.

\section{References}

1. Holmes B, Popoff M, Kiredjian M, Kersters K. Ochrobactrum anthropi gen. nov. from human clinical specimens and previously known as Group Vd. International Journal of Systematic Bacteriology 1988;38:406-16.

2. Ezzedine H, Mourad M, Van Ossel C, et al. An outbreak of Ochrobactrum anthropi bacteraemia in five organ transplant patients. $\mathrm{J}$ Hosp Infect 1994;27:35-42.

3. Christenson JC, Pavia AT, Seskin K, et al. Meningitis due to Ochrobactrum anthropi: an emerging nosocomial pathogen. A report of 3 cases. Pediatr Neurosurg 1997;27:218-21.

4. Vaidya SA, Citron DM, Fine MB, et al. Pelvic abscess due to Ochrobactrum intermedium [corrected] in an immunocompetent host: case report and review of the literature. J Clin Microbiol 2006;44:1184-6.

5. Alnor D, Frimodt-Moller N, Espersen F, Frederiksen W. Infections with the unusual human pathogens Agrobacterium species and Ochrobactrum anthropi. Clin Infect Dis
1994;18:914-20.

6. Elsaghir AA, James EA. Misidentification of Brucella melitensis as Ochrobactrum anthropi by API 20NE. J Med Microbiol 2003; 52:441-2.

7. Appelbaum PC, Campbell DB. Pancreatic abscess associated with Achromobacter group Vd biovar 1. J Clin Microbiol 1980; 12:282-3.

8. Mahmood MS, Sarwari AR, Khan MA, et al. Infective endocarditis and septic embolization with Ochrobactrum anthropi: case report and review of literature. J Infect 2000;40:28790 .

9. Ozdemir D, Soypacaci Z, Sahin I, et al. Ochrobactrum anthropi endocarditis and septic shock in a patient with no prosthetic valve or rheumatic heart disease: case report and review of the literature. Jpn J Infect Dis 2006;59:264-5.

10. Battaglia TC. Ochrobactrum anthropi Septic Arthritis of the Acromioclavicular Joint in an Immunocompetent 17 Year Old. Orthopedics 2008;31:606.

11. Barson WJ, Cromer BA, Marcon MJ. Puncture wound osteochondritis of the foot caused by CDC group Vd. J Clin Microbiol 1987;25:2014-6.

12. Wheen L, Taylor S, Godfrey K. Vertebral osteomyelitis due to Ochrobactrum anthropi. Intern Med J 2002;32:426-8.

13. Jelveh N, Cunha BA. Ochrobactrum anthropi bacteremia. Heart Lung 1999;28:145-6.

14. Higgins CS, Avison MB, Jamieson L, et al. Characterization, cloning and sequence analysis of the inducible Ochrobactrum anthropi AmpC beta-lactamase. J Antimicrob Chemother 2001;47:745-54.

15. Teyssier C, Marchandin H, Jean-Pierre $\mathrm{H}$, et al. Molecular and phenotypic features for identification of the opportunistic pathogens Ochrobactrum spp. J Med Microbiol 2005;54: 945-53.

16. Kern WV, Oethinger M, Kaufhold A, et al. Ochrobactrum anthropi bacteremia: report of four cases and short review. Infection 1993;21:306-10.

17. Daxboeck F, Zitta S, Assadian 0, et al. Ochrobactrum anthropi bloodstream infection complicating hemodialysis. Am J Kidney Dis 2002;40:E17. 